\title{
Metastable Aluminum Atoms Floating on the Surface of Helium Nanodroplets
}

\author{
Jay Jeffs, ${ }^{1}$ Nicholas A. Besley, ${ }^{1}$ Anthony J. Stace, ${ }^{1, *}$ Gautam Sarma, ${ }^{2}$ Ethan M. Cunningham, ${ }^{2}$ Adrian Boatwright, ${ }^{2}$ \\ Shengfu Yang, ${ }^{2}$ and Andrew M. Ellis ${ }^{2}$ \\ ${ }^{1}$ Department of Physical and Theoretical Chemistry, School of Chemistry, The University of Nottingham, \\ University Park, Nottingham NG7 2RD, United Kingdom \\ ${ }^{2}$ Department of Chemistry, University of Leicester, Leicester LE1 7RH, United Kingdom
}

(Received 25 November 2014; published 11 June 2015)

\begin{abstract}
Metal atoms have proved to be sensitive probes of the properties of superfluid helium nanodroplets. To date, all experiments on the doping of helium droplets have concentrated on the attachment of metal atoms in their ground electronic states. Here we report the first examples of metal atoms in excited states becoming attached to helium nanodroplets. The atoms in question are aluminum, and they have been generated by laser ablation in a metastable quartet state, which attaches to and remains on the surface of helium droplets. Evidence for a surface location comes from electronic spectra, which consist of very narrow absorption profiles that show very small spectral shifts. Supporting ab initio calculations show there to be an energy incentive for a metastable $\mathrm{Al}$ atom to remain on the surface of a helium droplet rather than move to the interior. The results suggest that helium droplets may provide a method for the capture and transport of metastable excited atomic and molecular species.
\end{abstract}

DOI: 10.1103/PhysRevLett.114.233401

PACS numbers: 36.40.Mr, 31.15.A-, 33.20.Lg

From their onset, experiments on doped helium nanodroplets have paid considerable attention to the spectroscopy of single atoms, since the associated shifts and widths of electronic spectral features have been found to be sensitive to whether an atom is located inside or on the surface of a droplet [1-4]. There appears to be general agreement that alkali metal atoms reside on the surfaces of droplets $[5,6]$, as do several of the alkaline earth metals [7-13]. Almost all such studies have concentrated on metal atoms with either no or one unpaired electron, and these have been added to the droplets in their electronic ground state; the only higher spin systems that have been investigated are chromium atoms, weakly bound alkali dimers and trimers, and the silver dimer [14-18].

Reported here are results from experiments where aluminum atoms have been ablated by laser from a solid sample of the metal in the presence of helium nanodroplets. From these experiments the presence of metastable aluminum atoms in the ${ }^{4} P\left(3 s 3 p^{2}\right)$ state at $\sim 29000 \mathrm{~cm}^{-1}$ above the ground state [19-21] has been established through observation of a series of 13 separate electronic transitions originating from this electronic state. In complete contrast to almost all previous studies, the wavelengths at which spectral transitions are recorded for these metal atoms in helium droplets exhibit almost no shift from those measured in the gas phase [19]. Transitions involving the metastable ${ }^{4} P$ states also exhibit comparatively narrow linewidths, which is again very unusual for helium nanodroplets. Narrow linewidths have been reported previously $[14,22]$ and in some cases interpreted as being due to a very weak interaction between the metal atom and the surrounding helium atoms [22].
There has been one previous study of the electronic transitions of aluminum atoms in helium droplets, by Reho et al. [23]. These authors identified two very broad absorption features (FWHM 420 $\mathrm{cm}^{-1}$ ) that were assigned to transitions from the ${ }^{2} P$ ground state to the lowest ${ }^{2} D$ excited state, and the authors concluded that spin-orbit coupling in the ${ }^{2} D$ state is quenched by the helium [23]. Previous observations of the spectra of alkali atoms in bulk helium have been interpreted in terms of a mechanism whereby excited spin-orbit states undergo rapid nonradiative relaxation to the lowest $(\Omega=1 / 2)$ level [24]. In contrast, the spectra presented here involve a number of spin-orbit states of electronically excited $\mathrm{Al}$ and there is no evidence of quenching. With the current high level of interest in both Rydberg atoms and their coherent control and the physics of ultracold atoms, state selectivity on the part of helium nanodroplets may provide a mechanism for generating and transporting excited atomic species.

The two instruments employed in these experiments use very similar methods to prepare helium droplets, but differ with regard to the technique used to record photoionization signals from $\mathrm{Al}$ atoms. Figure 1 shows a generic overview of the apparatus, and further details of how each experiment has been undertaken can be found in the Supplemental Material [25]. Photoionization spectra between 42000 and $44000 \mathrm{~cm}^{-1}$ have been recorded by monitoring $\mathrm{Al}^{+}$ signals as a function of laser wavelength following metal ablation in three separate regions of the apparatus. Although Fig. 1 shows the metal rod located between the first and second skimmers, denoted as region 2, experiments have also been undertaken with the rod located at positions 1 and 3. To establish which electronic states are 


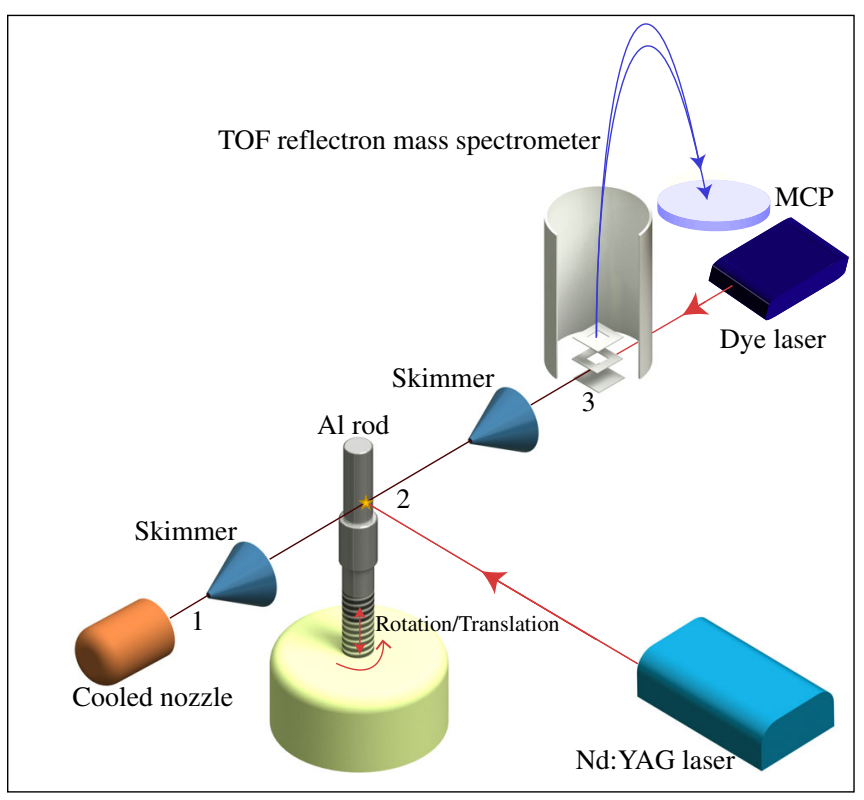

FIG. 1 (color online). Generic diagram that combines the essential features of the two separate pieces of apparatus used. Regions associated with the three separate experiments undertaken are labeled and the position of the rod moved accordingly.

being produced during laser ablation, Fig. 2(a) shows a spectrum recorded from isolated $\mathrm{Al}$ atoms ionized under gas phase conditions in the ion source of the reflectron (region 3 in Fig. 1). Figure 2(b) shows the same scan range,

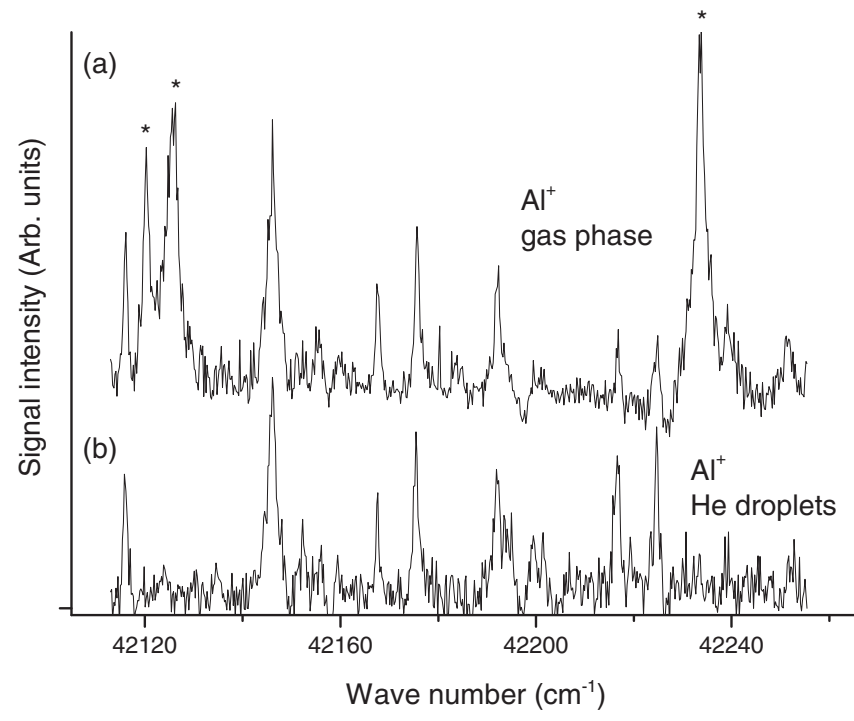

FIG. 2. (a) Photoionization spectrum recorded from gas phase ablated $\mathrm{Al}$ atoms (region 3 of Fig. 1). Transitions arising from nonresonant two-photon ionization of ground-state atoms are denoted with an asterisk. (b) Photoionization spectrum recorded from $\mathrm{Al}$ atoms ablated into the expansion region of the cooled nozzle (region 1 in Fig. 1 where helium droplets containing $10^{3}-10^{4}$ helium atoms were being formed). Both spectra were recorded by monitoring the $\mathrm{Al}^{+}$signal in the reflectron, and the gas phase spectrum has been shift upwards to avoid any overlap. but recorded from $\mathrm{Al}$ atoms ablated in the expansion region of the cooled nozzle (region 1 in Fig. 1 where helium droplets containing $10^{3}-10^{4}$ helium atoms were being formed). Note that the three transitions denoted by asterisks in Fig. 2(a) at $\sim 42120$ and $\sim 42230 \mathrm{~cm}^{-1}$, which are known to originate from aluminum atoms in the ground state [19], disappear in Fig. 2(b) due to spectral shifts identified by Reho et al. when $\mathrm{Al}$ atoms are embedded in helium droplets [23]. With the latter transitions omitted, two separate scans between 42000 and $44000 \mathrm{~cm}^{-1}$ show a total of 13 transitions associated with $\mathrm{Al}$ ablated in region 1 (see Fig. S5 of the Supplemental Material for a complete scan [25]). A search of known electronic transitions in aluminum atoms that, with a single photon, would place atoms above the lowest ionization limit $\left(48278.37 \mathrm{~cm}^{-1}\right)$ at the energies covered [19-21] suggests that all of the transitions shown in Fig. 2(b) originate from spin-orbit substates derived from the $\mathrm{Al}{ }^{4} P\left(3 s 3 p^{2}\right)$ state, which lies approximately $29000 \mathrm{~cm}^{-1}$ above the ground state. This state of $\mathrm{Al}$ is metastable because transitions to the ground electronic state are forbidden by both orbital and spin angular momentum selection rules, and thus a relatively long radiative lifetime is anticipated. One-photon transitions out of the ${ }^{4} P$ state would place the atoms in Rydberg states lying at $\sim 71000 \mathrm{~cm}^{-1}$, from where they could undergo autoionization. Similar sharp features seen in the UV spectra of sputtered silver atoms have been attributed to one-photon excitation from a metastable state of $\mathrm{Ag}$ I to a series of Rydberg states that subsequently autoionized [33].

To verify that the strong $\mathrm{Al}^{+}$signals shown in Fig. 2(b) are associated with the helium droplets and are not from $\mathrm{Al}$ atoms becoming entrained in the gas flow, additional spectra were recorded on a second apparatus. Here Al atoms were ablated in the presence of a collimated beam of helium nanodroplets (region 2 in Fig. 1 where droplets contain $\sim 10^{4}$ atoms). What characterizes these measurements is that it has been possible to monitor the intensities of the adduct ions, $\mathrm{Al}^{+}(\mathrm{He})_{n}$, as a function of laser wavelength. These results are shown in Fig. 3, where a summation of the intensities of the $n=1-3$ signals is compared with measurements of the $\mathrm{Al}^{+}$signal recorded under identical circumstances. As can be seen, over the range selected $42100-42225 \mathrm{~cm}^{-1}$, there is an excellent match between the two spectra. Since the beam density in the ionization region is very low, there is no possibility of bare $\mathrm{Al}^{+}$ions undergoing the three-body collisions necessary to generate $\mathrm{Al}^{+}(\mathrm{He})_{n}$ complexes, thus demonstrating that the sharp spectral transitions are indeed due to aluminum atoms attached to helium nanodroplets. Table I lists the 13 transitions that have been identified as due to the metastable ${ }^{4} P\left(3 s 3 p^{2}\right)$ state together with their shifts with respect to the gas phase transition energies and their linewidths. The magnitudes of the shifts are all close to the grating or calibration errors, but there appears to be a 


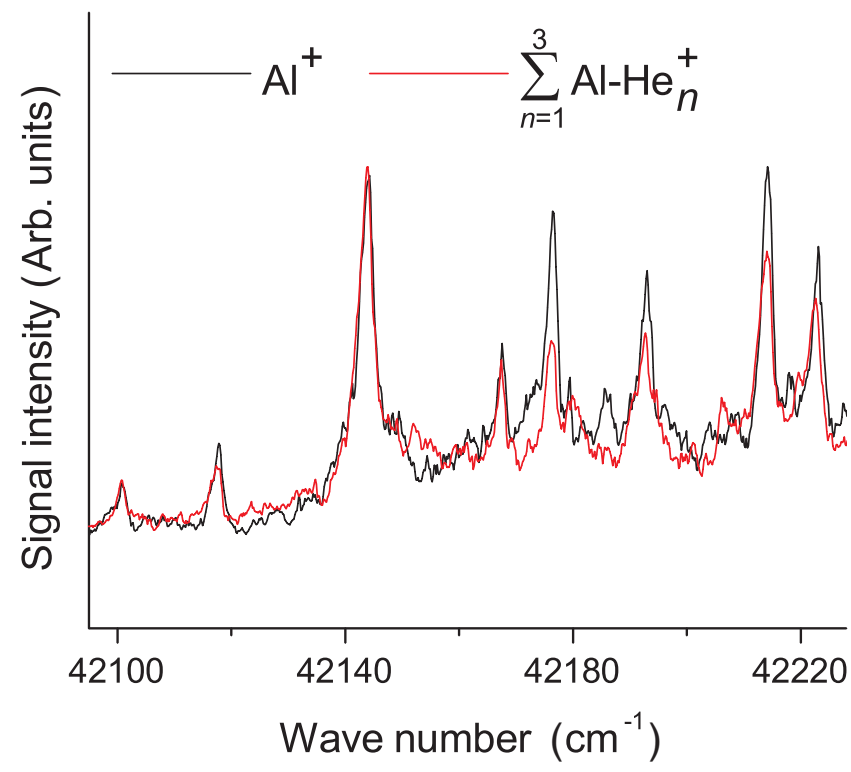

FIG. 3 (color). Photoionization spectra recorded following the ablation of $\mathrm{Al}$ atoms in the presence of a collimated beam of helium nanodroplets (region 2 in Fig. 1 where the droplets contain $\sim 10^{4}$ atoms). The spectra were recorded by monitoring the signal from either $\mathrm{Al}^{+}$(black line) or $\mathrm{AlHe}_{n}{ }^{+}$(by summation of the $n=1-3$ signals) (red line).

very small and consistent shift associated with the majority of transitions.

Table II shows the properties of potential energy curves calculated for $\mathrm{Al}-\mathrm{He}_{n}$ clusters using $\mathrm{CCSD}(\mathrm{T})$ (coupledcluster method with singles, doubles, and perturbative triples) methodology. Details of the calculations are in the Supplemental Material [25]. These calculations are for an aluminum atom in the ${ }^{4} P\left(3 s 3 p^{2}\right)$ state, which arises from the promotion of one of the electrons from the full $3 s$ orbital in the ground state to an unoccupied $3 p$ orbital in the ${ }^{4} P$ state. The key difference between this state and the ground electronic state is that there are now two occupied $3 p$ orbitals and one unpaired $3 s$ electron. In the $1{ }^{4} \Pi$ state of the $\mathrm{Al}-\mathrm{He}$ dimer there is an electron in the $3 p_{z}$ orbital pointing towards the helium atom and, as Table II shows, the resulting complex has a calculated binding energy of just $4.81 \mathrm{~cm}^{-1}$ and an internuclear separation $R_{\min }$ of $5.38 \AA$. In contrast, in the $1^{4} \Sigma$ state, the $3 p_{z}$ orbital is unoccupied and has a computed well depth of $173.9 \mathrm{~cm}^{-1}$. A similar pattern is observed for $\mathrm{Al}-\mathrm{He}_{2}$, where the binding energy per atom remains high. In the $\mathrm{Al}-\mathrm{He}_{4}$ complex the quartet state will necessarily have at least one occupied $3 p$ orbital orientated towards a helium atom. Similarly, for $\mathrm{Al}-\mathrm{He}_{6}$ the two atoms coordinating in the $z$ direction are again strongly bound, while the remaining four atoms bind more weakly. Overall, a consequence of increasing coordination is that the high binding energies seen for the smaller complexes start to decline. However, for $\mathrm{Al}-\mathrm{He}_{6}$ the binding energy per helium atom is calculated to be $41.3 \mathrm{~cm}^{-1}$, which is still 4 times larger than for the corresponding ${ }^{2} P$ electronic ground state [23]. In a final calculation, the preferred coordination of ground-and excited-state atoms has been compared in a cluster consisting of 98 helium atoms. From the results shown in Table II it can be seen that ground-state aluminum prefers, by a very small margin, to be at the center of a droplet, whereas the ${ }^{4} P$ excited state favors a surface site by the larger margin of $83 \mathrm{~cm}^{-1}$. A significant fraction of this additional stability comes from a strong association between ${ }^{4} P$ aluminum and a single helium atom. Optimized structures for the clusters with ground- and

TABLE I. Summary of electronic transitions recorded from the photoionization of metastable aluminum atoms in association with helium nanodroplets. All energies are in $\mathrm{cm}^{-1}$ and GP refers to gas phase.

\begin{tabular}{|c|c|c|c|c|c|c|c|c|}
\hline \multirow[t]{2}{*}{$\begin{array}{l}\text { Initial } \\
\text { state } \\
3 s 3 p^{2} \\
\end{array}$} & \multirow[t]{2}{*}{$\begin{array}{l}\text { Final state } \\
3 s 3 p\left({ }^{3} P^{0}\right) 3 d\end{array}$} & \multicolumn{2}{|c|}{$\begin{array}{l}\text { Angular momentum } \\
\text { states }\end{array}$} & \multirow[t]{2}{*}{$\begin{array}{l}\text { Initial GP } \\
\text { energy }\end{array}$} & \multirow[t]{2}{*}{$\begin{array}{c}\text { Final GP } \\
\text { energy }\end{array}$} & \multirow[t]{2}{*}{$\begin{array}{l}\text { He droplet } \\
\text { transition } \\
\text { energy }\end{array}$} & \multirow[t]{2}{*}{$\begin{array}{l}\text { Shift } \\
(\mathrm{He}-\mathrm{GP})\end{array}$} & \multirow[t]{2}{*}{$\begin{array}{c}\mathrm{He} \\
\text { linewidth }\end{array}$} \\
\hline & & $J_{i}$ & $J_{f}$ & & & & & \\
\hline${ }^{4} P$ & ${ }^{4} D^{o}$ & $1 / 2$ & $3 / 2$ & 29020.41 & 71244.17 & 42223.08 & -0.68 & 2.78 \\
\hline${ }^{4} P$ & ${ }^{4} D^{o}$ & $1 / 2$ & $1 / 2$ & 29020.41 & 71235.25 & 42214.21 & -0.63 & 2.91 \\
\hline${ }^{4} P$ & ${ }^{4} D^{o}$ & $3 / 2$ & $5 / 2$ & 29066.96 & 71260.55 & 42192.96 & -0.63 & 4.66 \\
\hline${ }^{4} P$ & ${ }^{4} D^{o}$ & $3 / 2$ & $3 / 2$ & 29066.96 & 71244.17 & 42176.51 & -0.70 & 1.78 \\
\hline${ }^{4} P$ & ${ }^{4} D^{o}$ & $3 / 2$ & $1 / 2$ & 29066.96 & 71235.25 & 42167.51 & -0.78 & 1.41 \\
\hline${ }^{4} P$ & ${ }^{4} D^{o}$ & $5 / 2$ & $7 / 2$ & 29142.78 & 71286.40 & 42143.83 & 0.21 & 2.27 \\
\hline${ }^{4} P$ & ${ }^{4} D^{o}$ & $5 / 2$ & $5 / 2$ & 29142.78 & 71260.55 & 42117.86 & 0.09 & 2.06 \\
\hline${ }^{4} P$ & ${ }^{4} D^{o}$ & $5 / 2$ & $3 / 2$ & 29142.78 & 71244.17 & 42100.78 & -0.61 & 1.35 \\
\hline${ }^{4} P$ & ${ }^{4} P^{o}$ & $5 / 2$ & $3 / 2$ & 29142.78 & 72250.53 & 43107.39 & -0.36 & 1.91 \\
\hline${ }^{4} P$ & ${ }^{4} P^{o}$ & $3 / 2$ & $3 / 2$ & 29066.96 & 72250.53 & 43181.88 & -1.69 & 1.65 \\
\hline${ }^{4} P$ & ${ }^{4} P^{o}$ & $3 / 2$ & $1 / 2$ & 29066.96 & 72277.75 & 43208.95 & -1.84 & 1.21 \\
\hline${ }^{4} P$ & ${ }^{4} P^{o}$ & $1 / 2$ & $3 / 2$ & 29020.41 & 72250.53 & 43226.71 & -3.41 & 1.23 \\
\hline${ }^{4} P$ & ${ }^{4} P^{o}$ & $1 / 2$ & $1 / 2$ & 29020.41 & 72277.75 & 43253.83 & -3.51 & 1.37 \\
\hline
\end{tabular}


TABLE II. Equilibrium internuclear separation $\left(R_{\min }\right)$ and well depth $(\varepsilon)$ for $\mathrm{Al}-\mathrm{He}_{n}$ clusters in the excited quartet state derived from $\operatorname{CCSD}(\mathrm{T}) / \mathrm{d}$-aug-cc-pVQZ calculations.

\begin{tabular}{|c|c|c|c|c|c|}
\hline Cluster & State & Al configuration & $R_{\min }(\AA)$ & $\varepsilon\left(\mathrm{cm}^{-1}\right)$ & $\varepsilon / n\left(\mathrm{~cm}^{-1}\right)$ \\
\hline $\mathrm{Al}-\mathrm{He}_{1}$ & $1{ }^{4} \Pi$ & $\begin{array}{l}{[\mathrm{Ne}] 3 s^{1} 3 p_{x}{ }^{1} p_{z}{ }^{1}} \\
{[\mathrm{Ne}] 3 s^{1} 3 p_{v}{ }^{1} p_{z}{ }^{1}}\end{array}$ & 5.38 & 4.8 & 4.8 \\
\hline \multirow[t]{3}{*}{$\mathrm{Al}-\mathrm{He}_{2}$} & $\begin{array}{l}1^{4} \Pi \\
1{ }^{4} \Pi_{u}\end{array}$ & $\begin{array}{l}{[\mathrm{Ne}] 3 s^{1} 3 p_{x}^{1}{ }^{1} p_{y}{ }^{1}} \\
{[\mathrm{Ne}] 3 s^{1} 3 p_{x}^{1} p_{z}{ }^{1}}\end{array}$ & 2.44 & 173.9 & 173.9 \\
\hline & & {$[\mathrm{Ne}] 3 s^{1} 3 p_{y}^{1} p_{z}^{1}$} & 5.35 & 8.6 & 4.3 \\
\hline & $1^{4} \Sigma_{u}^{-}$ & $\begin{array}{l}{[\mathrm{Ne}] 3 s^{1} 3 p_{x}^{1}{ }^{1} p_{y}{ }^{1}} \\
{[\mathrm{Ne}] 3 s^{1} 3 p_{x}{ }^{1} p^{1}}\end{array}$ & $\begin{array}{c}2.60 \\
2.65(2, z)\end{array}$ & 302.3 & 151.1 \\
\hline & & & $5.43(2, x)$ & 266.4 & 66.6 \\
\hline${ }^{\mathrm{a}} \mathrm{Al}-\mathrm{He}_{6}$ & & {$[\mathrm{Ne}] 3 s^{1} 3 p_{x}{ }^{1} p_{y}{ }^{1}$} & $\begin{array}{c}2.68(2, z) \\
5.39(4, x y)\end{array}$ & 247.7 & 41.3 \\
\hline${ }^{\mathrm{b}} \mathrm{Al}-\mathrm{He}_{98}$ & Center & {$[\mathrm{Ne}] 3 s^{2} 3 p^{1}$ g.s. ${ }^{\mathrm{c}}$} & 3.76 & 1293 & $\ldots$ \\
\hline $\mathrm{Al}-\mathrm{He}_{98}$ & Surface & {$[\mathrm{Ne}] 3 s^{2} 3 p^{1}$ g.s. } & 4.41 & 1285 & $\ldots$ \\
\hline $\mathrm{Al}-\mathrm{He}_{98}$ & Center & {$[\mathrm{Ne}] 3 s^{1} 3 p^{2}$ e.s. ${ }^{\mathrm{d}}$} & ${ }^{\mathrm{e}} 2.94$ & 1244 & $\ldots$ \\
\hline $\mathrm{Al}-\mathrm{He}_{98}$ & Surface & {$[\mathrm{Ne}] 3 s^{1} 3 p^{2}$ e.s. } & ${ }^{\mathrm{e}} 3.11$ & 1327 & $\ldots$ \\
\hline
\end{tabular}

${ }^{\mathrm{a}}$ The results for $\mathrm{Al}-\mathrm{He}_{6}$ have been derived from $\mathrm{CCSD}(\mathrm{T}) / \mathrm{d}$-aug-cc-pVTZ calculations.

${ }^{\mathrm{b}}$ All calculations on Al- $\mathrm{He}_{98}$ clusters are at the $\mathrm{RI}-\mathrm{MP} 2 / 6-31++G^{*}$ level of theory.

${ }^{\mathrm{c}}$ Ground state.

${ }^{\mathrm{d}}$ Excited state.

${ }^{\mathrm{e}}$ Most closely associated with a He single atom.

excited-state $\mathrm{Al}$ atoms are shown in Fig. 4. These results are significant for two reasons. First, the calculations show that in the experiment where excited-state atoms become attached to preformed helium droplets, the atoms will remain on the surface. Second, in the experiment where excited-state atoms are entrained in the helium expansion close to the nozzle (region 1 of Fig. 1), any aluminum atoms solvated in droplets will have an energy incentive to (a)

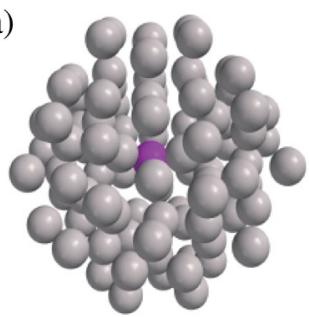

(c)

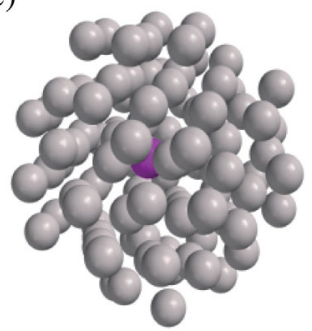

(b)

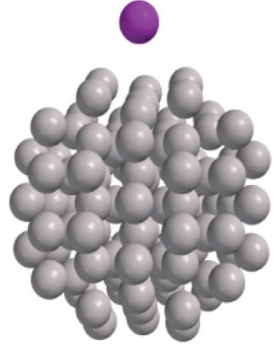

(d)

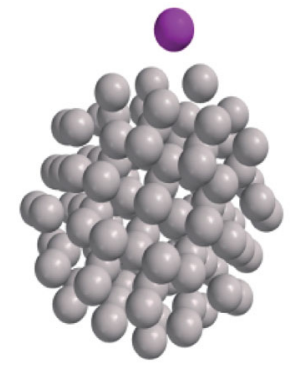

FIG. 4 (color online). Optimized structures calculated for an aluminum atom at the center and at the surface of a 98 atom helium cluster. (a),(b) $\mathrm{Al}$ in the electronic ground state. (c),(d) $3 s 3 p^{2}\left({ }^{4} P\right)$ excited state. move to the surface. Overall, the results show that the excited quartet state of the aluminum atom could occupy a comparatively stable site on or close to the surface of a helium droplet, where it would still be more strongly bound than solvated ground-state atoms.

The transitions identified in Table I involve all possible spin-orbit levels of the initial state, which implies a complete absence of any of the angular momentum relaxation mechanisms that have been discussed previously both for aluminum in helium droplets and for certain alkali metals in bulk helium $[23,24,34]$. The latter experiments have linked spin-orbit quenching to the formation of exciplexes, and metals with small spin-orbit splittings, such as $\mathrm{Li}$ and $\mathrm{Na}$, seem particularly susceptible [34]. In helium droplets the evidence is somewhat contradictory; surface-bound $\mathrm{Na}$ and $\mathrm{K}$ atoms not only form exciplexes with small numbers of helium atoms, but there is also an absence of spin-orbit quenching [35,36]. Excited-state metal-helium molecular orbitals associated with exciplex formation are exclusively of $\Pi$ symmetry, and excitation to any state with $\Sigma$ symmetry is assumed to result in desorption of the bare metal atom [37]. In contrast, the strongly bound dimer state identified here for the ${ }^{4} P \mathrm{Al}$ atoms has $\Sigma$ symmetry. There is the possibility that diatomic $\Pi$ state exciplexes involving the $3 s 3 p^{2}$ state are being expelled from the droplets and then photoionized. However, were that the case, then the subsequent excitation-autoionizing step might have been expected to yield vibrational structure, and that is not seen in either Fig. 2 or Fig. 3.

To account for the observation of the strong $\mathrm{Al}^{+}$signal after metastable excitation, the following sequence of 
events is proposed. First, $\mathrm{Al}$ atoms in the ${ }^{4} \mathrm{P}$ excited state are assumed to reside on the surface of helium droplets, which is strongly supported by the calculations presented here and by the experimental observation of narrow linewidths and negligible line shifts for spectroscopic transitions relative to the gas phase. Indeed, these observations suggest minimal interaction with the helium. Following photoexcitation, it is proposed that the resultant autoionizing state is expelled from the droplet prior to ionization, which would then ensure that predominantly $\mathrm{Al}^{+}$ions are formed in the process. A very similar sequence of events has been proposed by Federmann et al. [38] to account for the appearance of narrow absorption features in their Rydberg spectra of silver atoms trapped in helium droplets. However, unlike their system [38], the excited aluminum atoms reported here must undergo discrete excitation to an autoionizing Rydberg state before expulsion because the entire process requires just one rather than two photons.

UV photoionization spectra have been recorded from aluminum atoms in a metastable excited electronic state in the presence of a beam of helium nanodroplets, and where the observed transitions exhibit narrow linewidths and almost no spectral shift relative to the gas phase. Supporting $a b$ initio calculations show that there is an energy incentive for the excited state to reside on the surface of a droplet.

The authors would like to thank EPSRC and the Leverhulme Trust for financial support and Professor Tim Wright and Dr. Pascal Lablanquie for a number of helpful comments on the results. The Nuffield Foundation is also thanked for providing a bursary to E. M. C. Very helpful discussions with Professor W. H. Breckenridge on metal atom excited states are also acknowledged.

*tony.stace@nottingham.ac.uk

[1] K. B. Whaley, Int. Rev. Phys. Chem. 13, 41 (1994).

[2] J. P. Toennies and A. F. Vilesov, Annu. Rev. Phys. Chem. 49, 1 (1998).

[3] F. Stienkemeier and A. F. Vilesov, J. Chem. Phys. 115, 10119 (2001).

[4] J. Tiggesbäeumker and F. Stienkemeier, Phys. Chem. Chem. Phys. 9, 4748 (2007).

[5] F. Ancilotto, P. B. Lerner, and M. W. Cole, Low Temp. Phys. 101, 1123 (1995).

[6] F. Stienkemeier, J. Higgins, C. Callegari, S. I. Kanorsky, W. E. Ernst, and G. Scoles, Z. Phys. D 38, 253 (1996).

[7] F. Stienkemeier, F. Meier, and H. O. Lutz, J. Chem. Phys. 107, 10816 (1997).

[8] F. Stienkemeier, F. Meier, and H. O. Lutz, Eur. Phys. J. D 9 , 313 (1999).

[9] J. Reho, U. Merker, M. R. Radcliff, K. K. Lehmann, and G. Scoles, J. Chem. Phys. 112, 8409 (2000).

[10] A. Hernando, R. Pi, M. Mayol, M. Barranco, F. Ancilotto, O. Bunermann, and F. Stienkemeier, J. Phys. Chem. A 111, 7303 (2007).
[11] Y. Ren and V. V. Kresin, Phys. Rev. A 76, 043204 (2007).

[12] J. Navarro, D. Mateo, M. Barranco, and A. Sarsa, J. Chem. Phys. 136, 054301 (2012).

[13] F. Lackner, J. Poms, G. Krois, J. V. Pototshnig, and W. E. Ernst, J. Phys. Chem. A 117, 11866 (2013).

[14] M. Koch, A. Kautsch, F. Lackner, and W. E. Ernst, J. Phys. Chem. A 118, 8373 (2014).

[15] F. Stienkemeier, J. Higgins, W. E. Ernst, and G. Scoles, Phys. Rev. Lett. 74, 3592 (1995).

[16] J. Higgins, W. E. Ernst, C. Callegari, J. Reho, K. K. Lehmann, G. Scoles, and M. Gutowski, Phys. Rev. Lett. 77, 4532 (1996).

[17] J. Higgins, C. Callegari, J. Reho, F. Stienkemeier, W. E. Ernst, M. Gutowski, and G. Scoles, J. Phys. Chem. A 102, 4952 (1998).

[18] A. Przystawik, P. Radcliffe, S. Göde, K. H. Meiwesr-Broer, and J. Tiggesbäumker, J. Phys. B 39, S1183 (2006).

[19] http://www.nist.gov.

[20] K. Borje, K. B. S. Eriksson, and H. B. S. Isberg, Ark. Fys. 23, 527 (1963).

[21] V. Vujnović, K. Blagoev, C. Fürböck, T. Neger, and H. Jäger, Astron. Astrophys. 388, 704 (2002).

[22] A. Bartelt, J. D. Close, F. Federmann, K. Hoffmann, N. Quaas, and J. P. Toennies, Z. Phys. D 39, 1 (1997).

[23] J. H. Reho, U. Merker, M. R. Radcliff, K. K. Lehmann, and G. Scoles, J. Phys. Chem. A 104, 3620 (2000).

[24] T. Kinoshita, K. Fukuda, T. Matsuura, and T. Yabuzaki, Phys. Rev. A 53, 4054 (1996).

[25] See Supplemental Material at http://link.aps.org/ supplemental/10.1103/PhysRevLett.114.233401, which includes Refs. [26-32], for details of the apparatus, the experimental procedure, and the theoretical methods used to calculate $\mathrm{AlHe}_{n}$ cluster structures.

[26] A. Boatwright, N. A. Besley, S. Curtis, R. R. Wright, and A. J. Stace, J. Chem. Phys. 123, 021102 (2005).

[27] P. Claas, S.-O. Mende, and F. Stienkemeier, Rev. Sci. Instrum. 74, 4071 (2003).

[28] M. Mudrich, B. Forkl, S. Muller, M. Dvorak, O. Bunermann, and F. Stienkemeier, Rev. Sci. Instrum. 78, 103106 (2007).

[29] A. V. Kanaev, L. Museur, T. Laarmann, S. Monticone, M. C. Castex, K. von Haeften, and T. Moller, J. Chem. Phys. 115, 10248 (2001).

[30] D. E. Woon and T. H. Dunning,Jr., J. Chem. Phys. 98, 1358 (1993).

[31] H.-J. Werner et al., MOLPRO, version [2008]1, a package of ab initio programs, http://www.molpro.net.

[32] S. F. Boys and F. Bernardi, Mol. Phys. 19, 553 (1970).

[33] A. Wucher, W. Berthold, H. Oechsner, and K. Franzreb, Phys. Rev. A 49, 2188 (1994), and references therein.

[34] A. Hofer, P. Moroshkin, D. Nettels, S. Ulzega, and A. Weis, Phys. Rev. A 74, 032509 (2006).

[35] J. Reho, J. Higgins, C. Callegari, K. K. Lehmann, and G. Scoles, J. Chem. Phys. 113, 9686 (2000).

[36] J. Reho, J. Higgins, K. K. Lehmann, and G. Scoles, J. Chem. Phys. 113, 9694 (2000).

[37] C. P. Schulz, P. Claas, and F. Stienkemeier, Phys. Rev. Lett. 87, 153401 (2001).

[38] F. Federmann, K. Hoffmann, N. Quaas, and J. D. Close, Phys. Rev. Lett. 83, 2548 (1999). 